
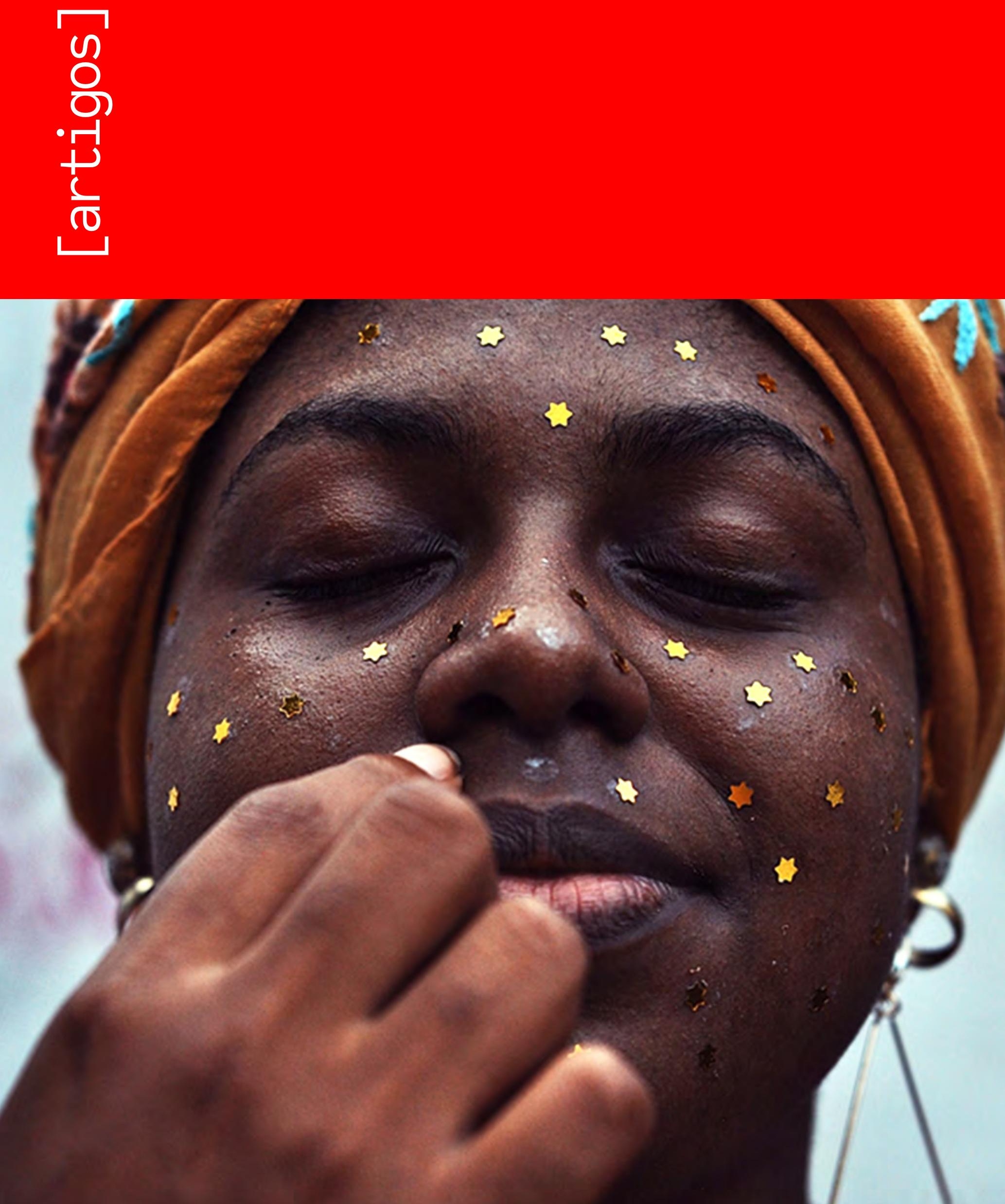


\section{Le corps de la mode. Histoire sociale de la mesure de l'Homme (Europe, 16e-19e siècle)}

O corpo da moda. História social da medida do homem (Europa, séculos XVI e XIX)

Fashion body. A social history of the measurement of Human (Europe, 16th-19th century)

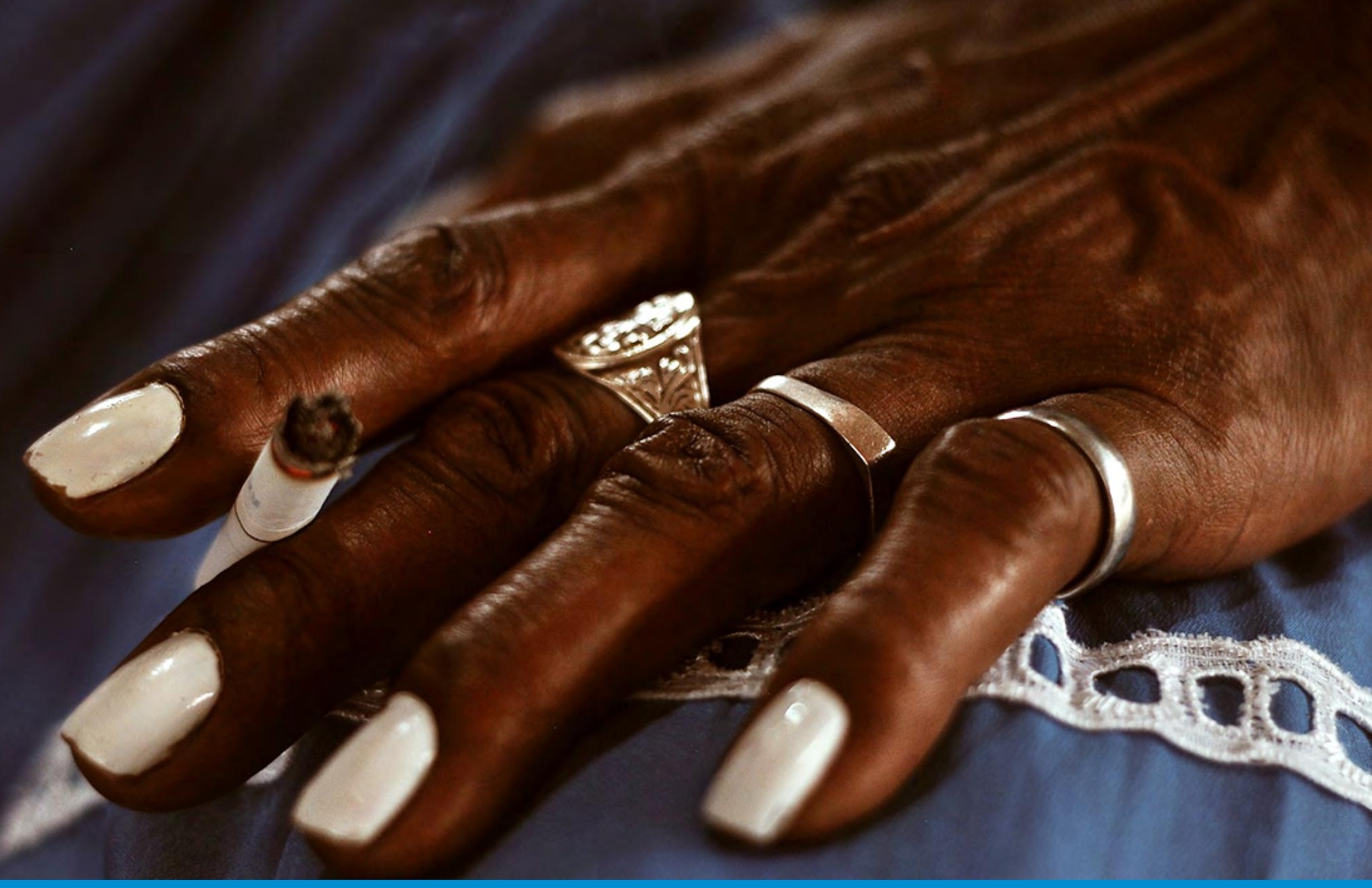




\section{Audrey Millet ${ }^{1}$}

ORCID: https://orcid.org/0000-0001-6163-0581

[résumé] De l'Homme de Vitruve à S, M et L du $19^{e}$ siècle, la mesure est au centre de la définition du corps humain et des modes. Sans mesures, pas de vêtement: le corps disparaît. L'enjeu de cet article est de proposer une série de questionnements pour comprendre la participation des praticiens du corps - du tailleur au perruquier - dans les changements de paradigmes imposant une nouvelle conception de l'Homme et un nouveau corps. L'histoire du corps et sa captation par les praticiens permet de compléter une vue de l'histoire de l'habillement : celle de la première enveloppe charnelle qui sert de support aux vêtements et aux artifices des apparences. Les transformations sociales et politiques du $19^{\mathrm{e}}$ siècle en Europe ont souvent été étudiées à partir des normes et des mesures coercitives. Néanmoins, je propose de renouveler les questionnements, et notamment d'examiner comment les savoirs corporels influencent le corps humain et nourrissent les réflexions depuis l'anthropométrie de la Renaissance jusqu'aux progrès technologiques de la première industrialisation. La fabrication des corps est un lieu d'excellence pour comprendre la dynamique de scientifisation qui touche l'Europe occidentale durant quatre siècles. Le corps est le moyen d'expression des normes économiques, sociales et politiques. À la croisée d'une histoire sociale des pratiques corporelles, de l'anthropologie des techniques et de l'épistémologie de l'esthétique, je propose d'interroger une histoire de la mesure de l'Homme à partir des savoirs des praticiens du corps.

[mots clefs] Corps. Savoirs. Vêtements. Techniciens. Mesures. Anthropométrie.

1 Audrey Millet est Docteure en histoire (Univers. Paris 8 et Neuchâtel). Ses articles et livres concernent l'histoire du travail, des savoir-faire et de l'industrie de l'habillement. Elle a publié Fabriquer le désir. Une histoire de la mode de l'Antiquité à nos jours (Paris: Belin, 2020). E-mail: audreypatrizia@yahoo.fr. 
[resumo] Do Homem Vitruviano ao homem P, M e G do século XIX, a medida está no centro da definição do corpo humano e das modas. Sem medidas, não há roupa: o corpo desaparece. 0 desafio deste artigo é propor uma série de questionamentos para compreender a participação dos profissionais que usam o corpo como suporte - do alfaiate ao peruqueiro - nas mudanças de paradigma, impondo uma nova concepção de homem e de corpo. A história do corpo e a sua captura pelos praticantes permitem completar uma visão da história do vestuário: a do primeiro envelope carnal que serve de suporte às vestimentas e aos artifícios das aparências. As transformações sociais e políticas do século XIX na Europa têm sido frequentemente estudadas com base em normas e medidas coercivas. No entanto, proponho renovar os questionamentos e, particularmente, examinar como os saberem influenciam o corpo humano e alimentam as reflexões desde a antropometria da Renascença até aos avanços tecnológicos da primeira industrialização. A fabricação de corpos é um lugar de excelência para compreender a dinâmica da cientificação que afetou a Europa Ocidental durante quatro séculos. 0 corpo é o meio de expressão das normas econômicas, sociais e políticas. No cruzamento de uma história social de realizações corporais, da antropologia das técnicas e da epistemologia da estética, proponho interrogar uma história da medida do homem a partir dos saberes dos praticantes do corpo.

\section{[palavras-chave] Corpo. Conhecimento. Vestuário. Técnicos. Medições. Antropometria.}

[abstract] From the Vitruvian Man to the S, M and L Human of the 19th century, measurement is at the center of the definition of the human body and fashions. Without measurements, no clothing: the body disappears. The challenge of this article is to propose a series of questions to understand the participation of body practitioners - from the tailor to the wigmaker - in the paradigm shifts imposing a new conception of Man and a new body. The history of the body and its capture by the practitioners allows to complete a view of the history of clothing: that of the first carnal envelope which serves as a support for clothing and the artifices of appearances. The social and political transformations of the 19th century in Europe have often been studied on the basis of norms and coercive measures. Nevertheless, I propose to renew the questioning, and in particular to examine how bodily knowledge influences the human body and nourishes reflections from the anthropometry of the Renaissance to the technological progress of the first industrialization. The manufacture of bodies is a place of excellence for understanding the dynamics of scientification that affected Western Europe for four centuries. The body is the means of expression of economic, social and political norms. At the crossroads of a social history of bodily practices, the anthropology of techniques and the epistemology of aesthetics, I propose to question a history of the measurement of Man based on the knowledge of practitioners of the body.

[keywords] Body. Knowledge. Clothing. Technicians. Measurements. Anthropometry.

Recebido em: 26-06-2020

Aprovado em: 22-07-2020 


\section{Etat des lieux et exposition de la démarche}

De l'Homme de Vitruve à l'Homme S, M et L du 19' siècle, la mesure est au centre de la définition du corps humain et des modes. L'enjeu de cet article est programmatique. Il propose une série de questionnements pour comprendre la participation des praticiens du corps - du tailleur au chirurgien - dans les changements de paradigmes imposant une nouvelle conception de l'Homme et un nouveau corps. Les transformations sociales et politiques du $19^{\mathrm{e}}$ siècle en Europe ont généralement été étudiées à partir des normes et des mesures coercitives. Néanmoins, je propose d'examiner comment les savoirs corporels influencent le corps humain et nourrissent les réflexions anthropométriques depuis la Renaissance jusqu'à la première industrialisation. La fabrication des corps est un lieu d'excellence pour comprendre la dynamique de scientifisation qui touche l'Europe occidentale durant quatre siècles. Le corps est le moyen d'expression des normes économiques, sociales et politiques. À la croisée d'une histoire sociale des pratiques corporelles, de l'anthropologie des techniques et de l'épistémologie de l'esthétique, je propose une histoire de la mesure de l'Homme à partir des savoirs des praticiens du corps. L'histoire du corps et sa captation par les praticiens permet de compléter une vie de l'histoire de la mode : celle de la première enveloppe charnelle qui sert de supports aux vêtements et aux artifices des apparences.

L'ambition est d'analyser les savoirs corporels comme une clef à la compréhension des normes et des standards qui se succèdent dans les sociétés occidentales. Il s'agit notamment de questionner les modalités d'incorporation des pratiques professionnelles pour comprendre la mise en place de grammaires des corps qui révèlent le coût d'acquisition de ce langage. Qui construit le corps? Quels sont ses savoirs ? De plus, il faut s'interroger sur la manière dont les normes corporelles émergent des pratiques artisanales et servent de nouveaux idéaux sociopolitiques. Le geste efficace permet de comprendre ce que les savoirs corporels disent de l'autonomie et de la dépendance des sociétés à l'égard de nouvelles normes puis de standards. La ligne de force de ce projet réside dans l'observation des techniques utilisées pour fabriquer les corps. De la « divine proportion » du $16^{\mathrm{e}}$ siècle à l'avènement des statistiques, il s'agit d'examiner les types de calculs jusqu'à la mise en place de moyennes et de procédures visant à définir un Homme neuf, standard caractéristique de l'essor des idées démocratiques et égalitaires. Ce projet peut être perçu comme une biographie des pratiques, miroir du calibrage des corps, de l'établissement d'une vérité scientifique définissant le corps « juste » et des normes sociétales. L'efficacité et l'impact du travail manuel sur les hiérarchies sociales et les contextes politiques méritent d'être examinés.

Cet article questionne l'histoire de la mesure du corps humain à partir d'un angle novateur, celui de l'apport des savoirs et savoir-faire corporels des praticiens - tailleurs d'habits, perruquiers, lunetiers, couturières - en Europe occidentale. Croisant corps et culture, Georges Vigarello, Philippe Perrot et Daniel Roche ont ouvert le champ à l'histoire des pratiques corporelles (VIGARELLO, 1987, 2004; PERROT, 1984; ROCHE, 1987). Mais l'analyse des enjeux sociaux et politiques de la transformation des corps à partir des techniques artisanales durant l'époque moderne a peu préoccupé les chercheurs. Si le $19^{e}$ siècle est généralement considéré comme celui de l'institutionnalisation de nouvelles disciplines, telle 
la statistique qui nourrit l'anthropométrie et la biométrie, il s'agit du résultat d'une dynamique de longue durée (NOIRIEL, 2007; DUBEY, 2007). C'est cette histoire de la mesure de l'Homme que je propose de questionner durant quatre siècles à partir des modifications des pratiques artisanales perceptibles dans les métiers du corps. Comment les normes corporelles, socio-esthétiques, émergent des pratiques artisanales?

Ma démarche s'articule autour de notions-clefs: les mesures, les normes, les praticiens et les savoirs corporels.

Mesures, car les étalons établissent des références de grandeur et/ou de poids caractéristiques du corps humain. En découlent des procédures d'évaluation qui définissent la modération du comportement et du mode de vie. Du $16^{\mathrm{e}}$ au $19^{\mathrm{e}}$ siècle, l'étalonnage, instable, nécessite des conversions qu'il s'agisse du système anthropomorphique, de la « divine proportion » ou du système métrique.

Normes, puisque les règles définissent un modèle courant ou une moyenne dégagée statistiquement qui représente les caractéristiques conformes à la majorité des corps. Elles induisent un ensemble de données - comme les mesures - qui justifient et imposent des jugements de valeur et règlent les existences sous peine de sanctions.

Praticiens, car ceux qui connaissent et exercent la pratique de techniques pour modifier, améliorer et calibrer les corps mettent en place des procédures de mesure de l'Homme. Les phénomènes microsociaux permettent de décrire des processus de normalisation et de comprendre la cohérence d'un système culturel, celui du corps, et d'un groupe, tailleurs ici, chirurgiens là - qui se construit à partir de pratiques.

Savoirs corporels, car les métiers étudiés ont une culture spécifique au corps humain - art de la coupe et de l'assemblage du vêtement, polissage du verre, montage des lunettes ou encore points de suture. Or, ces savoirs se modifient dans un contexte de scientifisation du monde du $16^{\mathrm{e}}$ au $19^{\mathrm{e}}$ siècle.

Dans les sociétés et États européens, la dissociation entre culture scientifique et technique oriente encore largement les questionnements sur les savoirs et les savoir-faire. Cette opposition appauvrit notre compréhension de tout un groupe de travailleurs qui participe par leurs techniques à définir l'« Homme pré-moderne et moderne ». Je propose d'exploiter une orientation peu présente dans les sciences sociales. Depuis la conception aristotélicienne, le travail, le monde de la matière et de la technique sont encore trop souvent considérés comme des champs mineurs (JACOMY, 1994). Les pratiques sont fréquemment éclipsées du champ des recherches. Pourtant, négliger ceux qui de la norme technique construisent les normes esthétiques - comme langage social - conduit à ignorer leur rôle dans l'affirmation de contraintes corporelles obéissant et définissant à un ordre social et politique. Tout un pan de l'activité humaine est alors marginalisé. Or, les pratiques et les savoirs professionnels ont une incidence directe sur la construction d'un « Homme moyen ». Ils pourraient éclairer les enjeux sociaux et politiques de l'identification physique des populations de la Renaissance à l'industrialisation. Les savoirs, lisibles dans les gestes, les outils et les résultats du travail, permettent de suivre les différentes conceptions du corps humain en adéquation avec l'essor des idées démocratiques et égalitaires vers 1800. Les praticiens, 
entendus comme groupe social et culturel, orientent bien la définition des normes et des anomalies. Les transformations des étalons, de la paume au pied, en passant par le mètre et les standards petit, moyen et grand, contribuent à la naissance de l'« Homme moyen ». Les procédures mises en place par les professionnels formatent les corps, dessinent les contours du normal et de l'anormal. Les savoirs corporels qui constituent une clef pour envisager les nouvelles procédures de mesure du corps humain, et le poids des normes et des standards sur la longue durée (NORTH, 1990). Ce projet peut être perçu comme une biographie des pratiques du corps dont le but serait de comprendre les changements de paradigmes et leurs conséquences sur la conception de l'Homme jusqu'à la mise en place de l'anthropologie judiciaire. L'analyse des savoirs et des techniques indiquera la mise en place de nouvelles procédures et des standards. Au centre de cette étude se situent les systèmes de mesure, des calculs mathématiques simples jusqu'à la mise en place de moyennes statistiques définissant le corps moyen. Les techniques et la rationalisation des pratiques permettent de comprendre ce que les savoirs disent de l'autonomie et de la dépendance des sociétés à l'égard des typologies corporelles. Je questionne le rôle des praticiens dans la production de codes sociétaux consolidés par l'émergence de nouveaux champs scientifiques. Mon projet se situe à la croisée de l'histoire sociale, de l'anthropologie des techniques et de l'épistémologie de l'esthétique.

De quelle manière les savoirs corporels des praticiens des apparences nourrissent l'anthropométrie de Quetelet et l'anthropométrie judiciaire de Bertillon ? Quand on évoque les faiseurs de vêtements, on semble bine loin de ces préoccupations. Pourtant, la découpe du corps, sa mesure ou son assemblage sont bien des savoirs transférables entre activités professionnelles. Caractéristique de la fin du $19^{\mathrm{e}}$ siècle, l'anthropométrie impose les moyennes statistiques comme socle des normes sociales. L'avènement de l'anthropométrie, c'est-à-dire d'une technique de mesure des particularités dimensionnelles de l'Homme (dimensions, masse, circonférences) impose une science de la mesure de l'Homme. Lorsque Bertillon s'en inspire et l'applique au système judiciaire, il met alors en place une technique qui repose sur l'analyse biométrique, c'est-à-dire un système d'identification à partir de mesures spécifiques, accompagné de photographies de face et de profil. Plutôt que de s'intéresser en premier lieu aux corps considérés comme anormaux ou déviants, je propose d'examiner les techniques de mesure utilisées pour fabriquer l'Homme moyen ou le « bon type ». $\mathrm{Du} 16^{\mathrm{e}}$ au $19^{\mathrm{e}}$ siècle, les corps de l'homme et de la femme révèlent les savoirs et les pratiques utiles à la fabrication du corps humain, miroir des normes et des standards, et l'apport des pratiques à la définition biopolitique de l'Homme normal (FOUCAULT, 2004). Je soupçonne les praticiens du corps d'avoir contribué à la stabilisation et à la convertibilité des mesures et d'avoir fourni les fondamentaux des systèmes de mesure nécessaires à l'homogénéisation d'un langage corporel chez les populations occidentales, notamment par le biais de la biométrie (BOURDIEU, 2004; CLOUSCARD, 1981; BARTHES, 1967). Ce lissage des systèmes de mesure et du corps humain pourrait être révélateur du coût d'acquisition d'un langage politique qui, sous couvert d'égalité entre les individus et d'ordre social, dépersonnalise les quotidiens et rejette les imperfections désormais nommées anomalies. Auparavant, les imperfections étaient acceptées et n'avaient ce sens si négatif que nos contemporains lui donnent. 
D’une part, je m'intéresserai à une relecture des savoirs qui permettra d'interroger l'«être professionnel » des praticiens, c'est-à-dire leurs connaissances et leurs compétences. Les livres techniques doivent ainsi être relus au prisme des pratiques afin de comprendre comment les praticiens eux-mêmes se perçoivent et veulent être perçus dans le cadre de métiers corporés ou libres. Ainsi, les modalités d'incorporation du métier se liront dans les trajectoires individuelles et collectives. Dans un deuxième temps, les transformations corporelles seront analysées en parallèle de l'essor des sciences du $16^{\mathrm{e}}$ au $19^{\mathrm{e}}$ siècle. L'examen des gestes indiquera l'utilisation des branches des mathématiques et de la médecine dans la fabrication d'un vêtement ou d'une couronne dentaire. Interroger la matérialité autorise ainsi à questionner la mise en place de systèmes de conversion, de mesures anthropométriques et du système métrique. La mesure devient alors un indicateur du passage de « artificiel- naturel » à l'Homme « normal-anormal ». Cette modification de conception mettra au jour des modifications intellectuelles, sociales et politiques basées sur le Juste Milieu, la moyenne. Or, ce Juste Milieu, les tailles $\mathrm{S}$, M et L, concorde avec le caractère restrictif des normes imposées par l'État moderne à des populations entières. Enfin, la technologie érigée en héroïne d'une société « avancée » s'analyse comme un des piliers de la tyrannie moderne. La construction de l'« Homme moyen » s'analyse à partir de l'imposition de nouveaux standards corporels révélateurs d'une politique qui rejette la bizarrerie, définit la déviance et exclut. La normalisation et l'exclusion des individus est en partie issue des techniques. La beauté du $19^{\text {e }}$ siècle, considérée comme laide par les contemporains, est avant tout la matérialisation d'un corps-statistique.

\section{Exposition de la démarche}

Ma proposition repose sur une hypothèse : la soumission des corps à la discipline sociale et la régulation politique des sociétés (THOMPSON, 1966) passent par les savoirs des praticiens qui favorisent l'émergence de sciences du corps. Ainsi, pour comprendre le « contrôle des sociétés », il s'agit d'examiner les savoirs techniques et théoriques des praticiens comme la clef d'une grammaire politico-esthétique (GALISON, 1997, p. 781-844).

S'intéresser aux savoirs corporels invite à reconsidérer les relations, les savoirs et savoir-faire à l'œuvre par l'exploration des métiers appliqués à modifier les corps. Des secteurs aussi variés que ceux de la mode, de l'optique et de la chirurgie, en apparence non connectés, sont à l'origine de changements durables. Dès le $16^{\mathrm{e}}$ siècle, l'essor de la culture consumériste, de matériaux moins onéreux, l'envie de posséder, de bien-être et de paraître accélèrent les transformations corporelles. En trouvant des solutions techniques pour améliorer et modifier les corps, les praticiens fabriquent un homme et une femme, miroir d'une vérité scientifique issue de la mesure. À partir de l'analyse des patrons, chiffres, comptabilités, dessins, vêtements et typologies physiques, je m'intéresserai à l' « être professionnel » des praticiens. J'entends par là examiner les modalités et le contenu de l'apprentissage, et de l'incorporation des savoirs (BOURDIEU, 2004). Les trajectoires individuelles et collectives témoignent de mutations (MILLET, 2015, 2018). Comprendre les savoirs corporels permettrait de saisir les étapes, non linéaires, de la mise en place de l'anthropométrie. Les hommes 
et les femmes des sociétés d'Europe occidentale adoptent au $19^{\mathrm{e}}$ siècle un vêtement standardisé. Il est le pendant civil de l'uniforme militaire qui le précède. Ce sont les tailleurs et les médecins qui unifient les premiers les systèmes de mesures corporelles afin de fabriquer un corps normalisé. La fabrication de standards n'émerge pas dans un premier temps du pouvoir politique mais des techniques de mesure et de la recherche d'efficacité des praticiens (EAMON, 2010; CONEIN, THÉVENOT, DODIER, 1993; SENNETT, 2008; CRAWFORD, 2009). L'étalonnage se situe au centre de la société. Il permet aux élites d'annoncer une vérité scientifique définissant « ce qu'il faut être » et de fabriquer la « déviance ». Or, le processus qui permet d'en faire une clef de lecture devrait être analysé durant quatre siècles à partir de ceux qui pratiquent la mesure des corps quotidiennement. Ma proposition entend donc s'intéresser à la fabrication de ces savoirs, non pas en étudiant les élites, mais par une « histoire d'en bas » (THOMPSON, 1966, p. 279-280).

Étudier les normes et les standards nécessite d'interroger ceux qui les créent matériellement. Ainsi, en croisant les savoirs et savoir-faire des praticiens du corps à une histoire de l'anthropométrie européenne, nous pourrions saisir les enjeux civiques d'un nouveau corps, celui de l'« Homme moyen ». Aussi, il s'agit de suivre les pratiques en marche de la Renaissance jusqu'à la première industrialisation. Néanmoins, je propose de réexaminer ce découpage chronologique qui fait passer subitement (KLEIN, SPARY, 2010), dans les années 1650, d'un monde ancien - artisanal - vers un monde moderne - scientifique - et nie l'apport continu des praticiens (SMITH, 2004). Les investigations se poursuivent donc jusque dans les années 1880 afin de comprendre comment un nouveau type d'Homme est adopté dans toute l'Europe.

De l'essor de l'alchimie à l'affirmation de l'anthropométrie, un vaste corpus regroupe sources manuscrites, littérature technique et objets. Les archives des corporations, actes des notaires (inventaires après-décès, liquidations de maîtrise, contrats d'apprentissage), dossiers de faillite, archives des écoles (écoles de dessin, académies de coiffure), dessins, esquisses et procès-verbaux de comparutions fournissent les indications indispensables pour préciser l'environnement économique et social des praticiens. La période est marquée par une inflation de la littérature technique. Environ 150 ouvrages sur les métiers (couture, perruque, chirurgie..) ont paru en espagnol, anglais, italien et allemand. Enfin, les vêtements, perruques, potions blanchissantes et lunettes, actes de la pratique, indiquent un souci d'économie, une rationalisation, les permanences et les transformations artisanales. L'approche méthodologique est à la fois quantitative et qualitative.

\section{Du naturel à la normalité: du corps-géométrique au corps-statistique}

Selon Habermas, le changement de terminologie du $16^{\mathrm{e}}$ au $19^{\mathrm{e}}$ siècle, notamment le passage du naturel au normal, va de pair avec une nouvelle approche de la scientificité (HABERMAS, 1992). L'essor des branches des mathématiques - algèbre, géométrie, statistique - et de la médecine appliqué au corps permet d'analyser la construction de l'homme moderne. La sémantique n'est qu'un indicateur du changement. En effet, les actes de la pratique complètent l'analyse des effets de ces transformations sur les concepts sociopolitiques. Les praticiens affirment « leur maîtrise des mathématiques pratiques » (VERIN, 1993) imposant 
ainsi la mesure comme un élément central de leurs savoirs durant toute la période. Je souhaite comprendre quel est le rôle de la mesure dans les étapes menant à la transformation d'un corps-géométrique à un corps-statistique.

\section{L'« Etre professionnel »: relire les savoirs}

De la Renaissance au $19^{\mathrm{e}}$ siècle, la distinction entre sciences intelligentes et sciences serviles issue de la tradition aristotélicienne nourrit les classifications qui conditionnent encore le regard du chercheur laissant la pratique dans une position inférieure. Mais pour comprendre le groupe des praticiens, il faut questionner les cadres de production des savoirs.

La littérature technique témoigne de l'émergence d'une nouvelle scientificité, plus rationnelle et mathématique du $16^{\mathrm{e}}$ au $19^{\mathrm{e}}$ siècle. Si elle n'est jamais attribuée aux praticiens, sa relecture témoigne d'un degré d'abstraction, une expérimentation contrôlée et instrumentée, une observation systématique, la régularité des enregistrements et l'usage d'outils mathématiques et statistiques. L'ambition des auteurs, c'est-à-dire l'abstraction revendiquée de pratiques, la « textualisation », est en partie responsable de la perte de contenu (DUBOURG-GLATINY, VÉRIN, 2004; PESTRE, 2015; KO, 2017). Georges Canguilhem explique les conséquences de la scientifisation de la société. « Décomposer, réduire, expliquer, identifier, mesurer, mettre en équations, ce doit bien être un bénéfice du côté de l'intelligence puisque, manifestement, c'est une perte pour la jouissance » (CANGUILHEM, 1952). Il est nécessaire de relire les pratiques résumées et abrégées, dépouillées du travail préparatoire, intellectuel et réflexif, pour comprendre l'action efficace, l'application des nombres et du calcul au corps humain. Les procédures de mesure jusqu'alors imperceptibles seraient enfin compréhensibles. Néanmoins, il faut également interroger les raisons du mépris à l'égard des pratiques artisanales. On le doit en partie au praticien lui-même qui tente de légitimer sa position sociale en se réclamant notamment des arts libéraux, seule catégorie lui permettant d'affirmer sa scientificité.

Dès le $16^{\mathrm{e}}$ siècle, les praticiens reprennent la rhétorique des ingénieurs. La perception qu'ils ont de leur propre statut s'analyse au prisme de l'essor de la consommation vestimentaire populaire, de la science oculaire ou d'une perception changeante des canons de beauté (VIGARELLO, 2004). Alors qu'au 16 ${ }^{\mathrm{e}}$ siècle, la « divine proportion » s'impose comme la référence des proportions humaines, trois siècles plus tard, le corps de asymétrique, voir bizarre, s'efface derrière trois tailles standards. Pour parvenir à définir des moyennes corporelles, les praticiens utilisent de nombreuses données scientifiques et complexifient leurs outils qui sont finalement repris par l'anthropologie judiciaire naissante. La capacité à mesurer devient alors le pendant de la scientificité des métiers de la pratique. Évaluation, justesse et précision définiraient le corps à adopter. Enfin, l'utilisation du même vocabulaire scientifique par les tailleurs ou les dentistes interroge sur le contenu de l'apprentissage. Il s'agirait donc de s'intéresser aux modalités de l'incorporation des savoirs en examinant les trajectoires individuelles et collectives.

Durant la période, les cadres de l'apprentissage peuvent être examinés à partir de la constitution du monde des praticiens, de ses champs et de ses secteurs. La famille ou les relations de proximité constituent une des bases de l'incorporation des gestes et savoirs du métier (BOURDIEU, 2004; BECKER, 1982). Les aspirants praticiens fréquentent de nombreux lieux : 
ateliers libres, écoles de dessin, des académies - de sciences ou de coiffure -, amphithéâtres anatomiques ou encore cours de dissection. Je propose donc d'interroger les modalités de formation au métier. Mais l'inflation d'informations (gravures, manuels et leçons publiques) n'a pas obligatoirement de conséquences sur les pratiques de mesure. Les connaissances des praticiens lisibles dans le résultat de leur travail (corps de jupe, polissage des verres de lunettes...) devraient, en revanche, fournir des informations pour comprendre le contenu des savoirs, les modalités de transmission et d'incorporation. Je dresserai donc des portraits individuels et de groupes afin de mettre en lumière leurs savoirs communs et d'autres plus spécifiques. L'analyse microhistorique des acteurs sert alors une biographie des pratiques qui, sur la longue durée, informe sur le changement de conception du corps humain en lien avec les variations de l'idéologie dominante.

\section{La mesure: des gestes à l’Homme}

Le geste normé doit être interrogé à partir du mouvement quotidien des membres du corps, du maniement des outils, des instruments et de la documentation. Il écourte l'action (MILLET, 2017). Le triomphe du geste se manifeste par la perfection croissante de l'ajustement du vêtement, des lunettes ou de la droiture du corps. Pour exceller, dans l'action, il faut crisper ses doigts sur l'ourlet, appliquer fermement sa main sur la peau... mais peu à peu l'approche directe et physique du client se transforme en maniement des chiffres. Les traces d'instruments conservées sur les vêtements dévoilent des classes d'opérations dont il s'agit de connaitre la part d'intuition ou de calculs (HILAIRE-PEREZ, 2013). " Prendre la mesure », « Tailler », « Ajuster », « Assembler et terminer le Corps », « Blanchir » nécessitent bien des opérations de calculs visant l'efficacité. Cette efficacité passe par la mise en place de processus dans le but de normaliser les pratiques. Dans quelle mesure la normalisation des gestes a-t-elle des conséquences sur la standardisation des corps ? Je propose d'interroger l'étendue de ces savoirs, des bases de calculs arithmétiques jusqu'aux règles complexes (BERNARD; CHAMBON; EHRHARDT; 2010).

Les bases du corps se modifient au $16^{\mathrm{e}}$ siècle lorsque les États occidentaux réaniment les canons classiques d'inspiration gréco-romaine. Vers 1490, Léonard de Vinci les applique aux dimensions physiques en s'inspirant de l'ingénieur romain Vitruve et notamment du livre 3 de son De Architectura (VITRUVE). L'« Homme est au centre de l'Univers » mais surtout au centre de la mesure. Son corps s'insère dans des cercles et carrés considérés comme les formes géométriques parfaites à la Renaissance. Vinci commente la conception du Romain « La Nature a distribué les mesures du corps humain comme ceci: Et quatre coudes font un double pas, et vingt- quatre paumes font un homme; et il a utilisé ces mesures dans ses constructions [...] ». Tous les membres du se répondent et forment une morphologie parfaite.

Pour venir à bout des aspérités d'une nature toujours perfectible - dents noires, corps trop gras ou trop maigre - les praticiens mesurent, tracent et réduisent en système pré-métrique. Dès le $16^{\mathrm{e}}$ siècle, les calculs se font systématiquement sous forme de fractions pour mesurer le tissu en largeur et longueur. À partir de l'unité de base, l'aune, la bara castillane ou la braccia florentine dont la longueur diffère d'un pays, d'une ville à l'autre, les praticiens utilisent les divisions en demi, tiers, quart, sixième, huitième ou plus. Sans unité de mesure 
homogène, la mondialisation de l'économie, l'accélération des modes et la circulation des biens obligent à maîtriser des tables de conversion bien connues au $18^{\mathrm{e}}$ siècle. La culture du chiffre sert également à la prévision des coûts (livres de compte, bons de commande). Les chiffres indiquent une topographie des corps fixée par des codes, points et zones essentiels. L'étude de ce langage permet de dévoiler des concepts opératoires nés de la pratique et de la capacité d'abstraction (HILAIRE-PEREZ, 2013). Cependant, un changement de paradigme apparaît autour de 1800. Imprécis et confus, les couples terminologiques « artificiel-naturel » et « anormal-normal » sont utilisés par diverses disciplines - psychiatrie, philosophie, sociologie ou anthropologie (DEVEREUX, 1957, p. 1-83; CANGUILHEM, 1943; FOUCAULT, 1999; DURKHEIM, 1993). Particulièrement utilisé chez les praticiens du corps, le vocable mérite d'être interrogé dans ses spécificités historiques pour éviter toute généralisation mal fondée ou anachronisme.

\section{De l'Homme naturel à l'Homme moyen}

L'Homme pré-moderne se définit autour des concepts de « naturel et d'artificiel », prédécesseurs binaires de « normal et anormal » mis en place au $19^{\mathrm{e}}$ siècle. Les premiers indiquent un comportement, c'est-à-dire qu'ils décrivent le corps et prescrivent une morale. En pratique, l'erreur de la nature est acceptée et peut être modifiée pour rentrer dans le rang grâce à l'artificiel. Quant aux concepts de« normal et d'anormal », forgés par la jeune discipline qu'est la psychiatrie, ils sont mesurables (DEVEREUX, 1957). En 1831, l'Oxford Dictionary donne la définition de « normal »: debout à l'angle droit. Celle-ci n'est plus morale mais indique une mathématisation, une évaluation et une description. La notion de mesure informe alors sur « ce qui est » et impose " ce qui doit être », c'est-à-dire une norme sociale (HABERMAS, 1996). Ce changement est directement issu de nouvelles perspectives techniques soutenues par l'essor des statistiques. Au 19e siècle, s'impose l' « Homme vrai » dont les dimensions sont tirées de moyennes. Dans les années 1830, le statisticien Adolphe Quetelet quantifie mathématiquement, extrapole les hauteurs, les poids, les largeurs, la circonférence des membres du corps moyen (QUETELET, 1835). La moyenne qui en ressort est la base des mesures de la majorité des Hommes. Le nouvel idéal devient alors une taille $S$, M ou L. Il est la manifestation physique d'un nouvel idéal politique, celui du Juste Milieu, et de l'essor des idées égalitaires en Europe occidentale. Le nouveau corps témoigne de l'oppression et du caractère restrictif des normes imposées par l'État moderne sur des groupes d'individus. Les modifications pratiques de l'art de mesurer sont caractéristiques des changements de paradigmes scientifiques mais aussi sociaux et politiques.

\section{La tyrannie de la mesure: quand la technologie crée les standards}

L'efficacité qui anime les sociétés européennes de l'époque moderne indique une économie des gestes dans l'intention fabricatrice des praticiens. Il vise à reformer/réformer les anatomies pour les rendre socialement acceptables. Cependant à l'aube du $19^{\mathrm{e}}$ siècle, les pratiques fabriquent un système d'exclusion. 


\section{De la norme au standard corporel: l'habit neuf du politique}

Utiliser des concepts clefs à diverses branches des sciences sociales, comme norme, normativité ou normalisation, est fertile pour se détacher des frontières disciplinaires trop étroites (DEVEREUX, 1957). Lorsque Durkheim, Canguilhem et Foucault prennent comme point de départ les conventions de comportement et les normes de valeur qui les définissent, déjà une distinction s'opère entre les normes sociales qui relèvent de l'autorité, et les normes statistiques attachées aux standards. Les directives normatives servent à contrôler ou intervenir sur le corps social. Cependant, les actes de la pratique apportent une règle ou une ligne à suivre. Mais lorsque la norme devient une règle naturelle d'accomplissement ou d'efficacité de plus en plus fréquemment calculée aux moyens de statistiques ou d'une procédure, elle se transforme alors en standard. Le standard limite bien plus le « non-conformisme normal » ou la bizarrerie que la norme (FOUCAULT, 1994). Il devient alors dangereux car c'est lui qui définit l'appartenance à un groupe ou son exclusion.

Lorsque les praticiens rassemblent puis ordonnent des données et des outils afin de mettre en place un raisonnement scientifique et systématique, ils produisent une procédure aboutie menant facilement à l'efficacité. Je propose d'explorer comment l'efficacité technique permet l'imposition du standard. La moyenne statistique appliquée au corps instaure une tyrannie de la mesure: elle rejette les corps dont les mesures ne correspondent pas à la majorité. Les procédures de mesure du corps sont normalisées et standardisées. Pourtant durant l'Ancien-Régime, la variation physique est seulement perçue comme personnelle. Elle n'exclut pas automatiquement. Au $19^{\mathrm{e}}$ siècle, au contraire, elle devient une déviation de la taille - c'est-à-dire de la norme absolue. L'hégémonie de la mesure statistique s'affirme comme un mode d'enquête scientifique et sociale, mais les recherches n'ont pas encore exploité les apports des praticiens dans ce domaine (CHIAPELLO; DEROSIERES, 2006; DESROSIERES, 1993). Pourtant, leurs procédures participent à l'essor de l'anthropométrie, discipline au double visage basée sur l'inclusion et l'exclusion (HACKING, 1975, 1990).

$\mathrm{Au}$ lieu de commencer par l'étude des normes, je propose d'analyser le travail technique en amont de la fabrication des corps, pour suivre les pratiques en marche et leurs rapports tendus avec la montée des idées démocratiques et égalitaires, et les principes normatifs des apparences en Europe. Selon Quetelet, « L'Homme moyen est dans une nation ce que le centre de gravité est au corps » (QUETELET, 1835). Je questionnerai l'articulation entre uniformité et besoins personnels d'une identité individuelle. Depuis le $17^{\mathrm{e}}$ siècle, la quête de l'exactitude porte les sciences. L'environnement commercial de plus en plus compétitif, la commercialisation, l'intérêt croissant pour l'innovation technologique et la course aux brevets participent à la définition d'une science du corps: la vérité scientifique exclue le hors-normes. Un nouveau type d'entrepreneurs utilise les idées de proportions, les tailles et les mesures pour fabriquer en série. Les pratiques traditionnelles ont participé à cette nouvelle conception du corps. Si au début du $19^{\mathrm{e}}$ siècle les mesures et la standardisation sont décriées, elles sont rapidement acceptées comme miroir des idées démocratiques et égalitaires naissantes. Les praticiens donnent une matérialité à la démocratisation de la société 
en effaçant les distinctions de classes perceptibles au premier coup d'œil. Le physique dit alors le comportement social comme le prouvent les travaux de Lavater à Galton (LAVATER, 1820). Les praticiens se mettent en ordre de bataille pour créer ce nouveau corps humain, social et politique, qui se construit dans un premier temps dans l'atelier.

\section{Une technologie qui normalise et exclut}

Durant l'Ancien-Régime, même si des modes s'imposent, au premier abord, chaque client a ses propres mesures, son vêtement, ses lunettes ou ses implants dentaires adaptés. Mais les vêtements des marins ou l'armée, tendent précocement à définir des normes absolues. Le système n'est pas entièrement basé sur les mesures personnelles. Les manuels et les outils indiquent une dynamique de rationalisation. En effet, le système est déjà mixte à la Renaissance, entre sur-mesure et fabrication en série, indiquant une fabrication flexible et qualitative. L'Homme moderne se dessine à travers typologies, disparition du contact physique direct avec le client et observation visuelle pour travailler les défauts et les écarts à la norme. Le basculement a lieu lors du passage du système de mesure anthropomorphe au mètre durant la Révolution française (SCHOFIELD; STRIKER, 1986; PORTER; TEICH, 1992; FRAISSE, 1994). Lavoisier et Condorcet établissent un mètre provisoire dès 1793, définitivement fixé six ans plus tard. Après les tailleurs militaires, les civils s'en inspirent et transforment ainsi le client mesuré en taille. Le mètre ruban flexible ou les décimètres s'invitent dans le bagage du tailleur ou du médecin vers 1800. Les idées égalitaires imposent un calibrage largement adopté en Europe occidentale mais dont il s'agit aussi d'analyser les modalités d'acceptation ou de rejet. Efficacité, construction et simplicité deviennent alors les maîtres mots du corps du $19^{\mathrm{e}}$ siècle. Le héros antique, Apollon du belvédère ou Vénus Médicis, est remplacé. Mètres et centimètres s'imposent comme des outils de valeur pour leur exactitude scientifique. La mesure permet alors de produire des statistiques et des moyennes. Les conséquences du nouveau système de mesure se traduisent par l'inflation des brevets et la complexification des instruments. L'ambition est de gagner en efficacité et en précision.

Dès les premières années du $19^{\mathrm{e}}$ siècle, les tailleurs brevètent des costumomètres ou des longimètres. Ils transforment alors l'approche qualitative et le langage mathématique $\mathrm{du} 18^{\mathrm{e}}$ siècle en exercice géométrique. Le corps est devenu une conjonction de parallélogrammes, de courbes doubles et de points pour produire un vêtement disponible dans un ensemble de tailles (INPI). La croissance du nombre de brevets, notamment validés par les institutions, témoigne de l'intérêt de l'industrie de l'habillement et des autorités pour la fabrication en série. Les praticiens homogénéisent les pratiques sociales en interrogeant l'art et la manière de mesurer. Durant les années 1830-40, le somatomètre indique une autre étape de la mesure des silhouettes. Le corps est emballé dans un instrument composé de règles souples qui se transforment en une armure définissant un squelette métallique. La cage encercle le corps du cou aux orteils mesurant aussi la circonférence des bras et des cuisses. L'Homme de mode est quantifié donnant ainsi un pouvoir à celui qui utilise l'instrumentation soutenue par un vocabulaire pseudo-scientifique stimulant la vente. Cependant, 
l'imposition des tailles $\mathrm{S}, \mathrm{M}$ et $\mathrm{L}$ a des conséquences sociales non négligeables. D'une part, elle modifie sur le long terme l'idéal de beauté et, d'autre part, elle exclut de fait toute une frange de la population.

\section{Une beauté statistique?}

$\mathrm{Au}$ tournant des $18^{\mathrm{e}}$ et $19^{\mathrm{e}}$ siècles, les bizarreries de l'être humain asymétrique (épaules inégales, jambes courtes...) deviennent des écarts et des déviances - en somme des anomalies (BREWARD, 2001; BECKER, 1963). Je propose d'interroger les corps dans une perspective relevant de l'expérience et du poststructuralisme. Alors que les praticiens sont les seuls capables d'intervenir sur les silhouettes, d'en dissimuler les asymétries et d'en flatter certaines difformités pour correspondre à la norme en vogue, l'avènement de moyen tend à limiter leur intervention. Le nouvel idéal de beauté est accusé de conditionner les corps par leur dépersonnalisation. Certains contemporains accusent les médias et la commercialisation à grande échelle d'en être responsables. De la diffusion de la gravure aux présentations des expositions universelles, les normes sont devenues une préoccupation constante, quotidienne et incontestable. En 1858, spectateur des modifications de la silhouette, Théophile Gautier cristallise les débats entre beau idéal et beau réel, beauté classique et laideur moderne. De la même manière les tailleurs se montrent réticents à abandonner leur grammaire vestimentaire qui différencie l'employé de bureau et le médecin jusqu'au $19^{\mathrm{e}}$ siècle. Le type moderne ne repose plus sur la perfection physique géométrique mais sur des calculs issus de statistiques systématiquement basées sur des mesures de corps réel. L'Homme moyen déteint sur le vêtement devenu tout aussi moyen. En creux, on perçoit dans les nouvelles pratiques, les effets de la modernité et, notamment de la culture urbaine qui augmente le nombre d'anomalies et entre en conflit avec les modèles classiques et médicaux. Ironiquement, les recherches des praticiens ouvrent la voie à la production à grande échelle de vêtements civils, aux modèles corporels et à la production de masse qui considère seulement trois types de corps acceptables - au dixième de décimale près (BREWARD, 2001; THESANDER, 1997). Ces tailles qui « conduisent à l'uniforme indifférent et absolu » sont censées gommer les individualités et le laid.

Les réflexions portant sur les mesures modifient sur le long terme le concept de corps unique et individuel. Les études de la mode pourraient s'inspirer de l'enfermement des corps pour nuancer, notamment, les concepts de personnalisation et d'individualisation. Avec l'apport des statistiques, le corps devient une unité quantifiable et comparable, force de stabilité et de cohésion d'une population. Les praticiens en définissant et imposant des moyennes participent alors à l'avènement de l'anthropométrie dans la seconde moitié du $19^{\mathrm{e}}$ siècle. Criminologues, anthropologues ou eugénistes, de Bertillon à Galton, les amateurs de l'anthropométrie utilisent les statistiques comme stratégies de représentations et de mesures du corps humains (HARTLEY, 2001). Ils définissent des modèles et cataloguent des typologies de corps, normalisant, créant des taxinomies, classant et classifiant les caractères physiques et les défauts. Ces systèmes sont basés sur le principe de l'existence de standards 
et de normes, excluent les anomalies devenues incompatibles avec une nouvelle conception du corps social et politique dans un contexte où le réel est considéré comme laid, voire non viable. Les pratiques, qu'il faut également interroger au prisme du genre, visent à mettre au jour l'« moyen », fondement de l'anthropologie scientifique qui se confond avec le corps à la mode imposé par les tendances.

\section{Références}

BECKER, Howard. Art worlds. Berkeley, London: University of California Press, (1982) 2010.

BERNARD, Alain; CHAMBON, Grégory; EHRHARDT, Caroline. Le sens des nombres. Mesures, valeurs chiffrées, représentations de grandeurs réelles. Une approche historique. Paris: Vuibert, 2009.

BOAS, Franz. Anthropology and modern life. London: George Allen \& Unwin, 1929.

BOURDIEU, Pierre. Esquisse pour une auto-analyse. Paris: Raison d’Agir, 2004.

BARTHES, Roland. Système de la mode. Paris: Seuil, 1967.

BREWARD, Christopher. Manliness, modernity and the shaping of male clothing. In: ENTWISTLE, Joanne; WILSON Elisabeth (edits.). Body dressing. Oxford: Berg, 2001, p. 175.

BECKER, Howard. Outsiders. Études de sociologie de la déviance. Paris: Métailié, (1963) 1985.

THESANDER, Marianne. The feminine ideal. London: Reaktion, 1997.

CANGUILHEM, Georges. La connaissance de la vie. Paris: Vrin, (1952) 1992.

CANGUILHEM, Georges. Le normal et le pathologique. Paris: PUF, (1943) 2015.

CERUTTI, Simona. Who is below?. E. P. Thompson, historien des sociétés modernes: une relecture. Annales. Histoire, Sciences Sociales, v. 70, n. 4, 2015, p. 932-933. Disponible en: https://www.cairn.info/revue-annales-2015-4-page-931.htm\#. Accès en: 18 octobre 2020.

CHIAPELLO, Eve; DESROSIÈRES, Alain. La quantification de l'économie et la recherche en sciences sociales: paradoxes, contradictions et omissions. Le cas exemplaire de la positive accounting theory. In: EYMARD-DUVERNAY, François (dir.) L'Économie des conventions. Paris: La Découverte, 2006, t. 1, p. 297-310. 
CLOUSCARD, Michel. Le capitalisme de séduction. Critique de la social-démocratie libertaire. Paris: Ed. Sociales, 1981.

CONEIN, Bernard; DODIER, Nicolas; THÉVENOT, Laurent (edits.). Raisons pratiques. Les objets dans l'action. Paris: Ed. EHESS, 1993, v. 4.

INPI. Institut National de la Propriété Industrielle. Brevets $19^{\mathrm{e}}$ siècle.

CRAWFORD, Matthew. Shop class as soulcraft: An inquiry into the value of work. New York: Penguin, 2009.

DESROSIÈRES, Alain. La politique des grands nombres. Histoire de la raison statistique. Paris: La Découverte, (1993) 2000.

DEVEREUX, Georges. Essai d'ethnopsychiatrie générale. Paris: Gallimard, (1957) 1970, p. 1-83.

DUBEY, Gérard. Les deux corps de la biométrie. Communications. Corps et techniques, $\mathrm{n}$. 81, 2007, p. 153-166. Disponible en: https://www.persee.fr/doc/comm_0588-8018_2007_ num_81_1_2465. Accès en: 18 octobre 2020.

DUBOURG-GLATINY, Pascal, VÉRIN Hélène. Réduire en art, la technologie de la Renaissance aux Lumières. Paris, MSH, 2008.

DURKHEIM, Èmile. The normal and the pathological. In: PONTELL, Henry N. (edits.). Social Deviance Readings in Theory and Research. Upper Saddle River: Prentice Hall, 1993.

EAMON, William. The professor of secrets: mystery, medicine, and alchemy in renaissance Italy. Washington: Washington University Press, 2010.

FOUCAULT, Michel. Dits et écrits. Paris: Gallimard, 1994.

FOUCAULT, Michel. Les anormaux: cours du Collège de France. Paris: Gallimard/Seuil, 1999.

FOUCAULT, Michel. Naissance de la biopolitique, cours au Collège de France 1978-1979. Paris: Gallimard Seuil, 2004.

FRAISSE, Geneviève. Reason's muse: sexual difference and the birth of democracy. Chicago: University of Chicago Press, 1994.

GALISON, Peter. Image and logic: a material culture of microphysics. Chicago: University Chicago Press, 1997, p. 781-844. 
GALTON, Francis. Regression towards mediocrity in hereditary stature. Journal of the Anthropological Institute, v. 15, 1886, p. 246-263. Disponible en: http://www.stat.ucla. edu/ nchristo/statistics100C/history_regression.pdf. Accès : 20 octobre 2020

HABERMAS, Jürgen. Between facts and norms. Contributions to a discourse theory of law and democraty. Cambridge: MIT, (1992) 1996.

HACKING, Ian. The emergence of probability: a philosophical study of early ideas about probability, induction and statistical inference. Cambridge: Cambridge University Press, (1975) 2003.

HACKING, Ian. The taming of chance. Cambridge: Cambridge University Press, 1990.

HARTLEY, Lucy. Physiognomy and the meaning of expression in Nineteenth-Century culture. Cambridge: Cambridge University Press, 2001.

HILAIRE-PÉREZ, Liliane. La pièce et le geste. Artisans, marchands et savoir technique à Londres au 18 ${ }^{\mathrm{e}}$ siècle. Paris: Albin Michel, 2013.

JACOMY, Bruno. Le faire savoir des savoir-faire. In: Bayle François (dir.). L'Empire des techniques. Paris: Le Seuil, 1994, p. 48-49.

KLEIN, Ursula; SPARY, Emma C. Materials and expertise in early modern Europe: between market and laboratory. Chicago: University of Chicago Press, 2010.

LAVATER, Johann Kaspar. L'Art de connaître les hommes par la physionomie. Paris: Levrault, Shoell et Cie, 1806.

MILLET, Audrey. Factory Draughtsmen in Eighteenth and Nineteenth-Century France. The Forgotten Artists of the Workshop. Symbolics Goods. Artistes ordinaires: du paradoxe au paradigme?, n. 1, 2017. Disponible en: https://www.biens-symboliques.net/106. Accès en : 18 octobre 2020.

MILLET, Audrey. Vie et destin d'un dessinateur textile. D'après le journal d'Henri Lebert (1794-1862). Seyssel: Champ Vallon, 2018.

NOIRIEL, Gérard. L'identification. Genèse d'un travail d'État. Paris: Belin, 2007.

NORTH, Douglass. Institutions, institutional change and economic performance. Cambridge: Cambridge University Press,1990.

PERROT, Philippe. Le travail des apparences ou Les transformations du corps féminin: XVIII ${ }^{\mathrm{e}}$-XIX ${ }^{\mathrm{e}}$ siècle. Paris: Le Seuil, 1984. 
PESTRE, Dominique (dir.). Histoire des sciences et des savoirs. Paris: Seuil, 2015, t. 1.

KO Dorothy. The social life of inkstones. Artisans and scholars in Early Qing China. Chicago: University of Washington Press, 2017.

PORTER, Roy; TEICH Mikulàs (edits.). The Scientific Revolution in national context. Cambridge: Cambridge University Press, 1992.

QUETELET, Adolphe. Sur l'homme et le développement de ses facultés ou Essai de physique sociale. Paris: Bachelier, 1835.

ROCHE, Daniel. La culture des apparences. Une histoire du vêtement (XVIIe-XVIIIe siècles). Paris: Fayard, 1989.

SCHOFIELD, Malcolm; STRIKER, Gisela (edits.). The norms of nature: studies in hellenistic ethics. Cambridge: Cambridge University Press, 1986.

SENNETT, Richard. The craftsman. New Haven: Yale University Press, 2008.

SMITH, Pamela. The body of the artisan. Art and experience in the Scientific Revolution. Chicago: University of Chicago Press, 2004.

KOYRÉ, Alexandre. Du monde clos à l'univers infini. Paris: PUF, (1957) 1962.

THOMPSON, Edward P. The making of the english working class. Toronto: Penguin Books, (1966) 1991.

VÉRIN, Hélène. La gloire des ingénieurs. L'intelligence technique du $16^{\mathrm{e}}$ au $18^{\mathrm{e}}$ siècle. Paris: Albin Michel, 1993.

VIGARELLO, Georges. Le propre et le sale: l'hygiène du corps depuis le Moyen Âge. Paris: Le Seuil, 1987.

VIGARELLO, Georges. Histoire de la beauté: le corps et l'art d'embellir de la Renaissance à nos jours. Paris: Le Seuil, 2004. 